\title{
A GEOMETRIC MEANING OF THE RANK OF HERMITIAN SYMMETRIC SPACES
}

\author{
Dedicated to Professor I. Mogi on his 60th birthday \\ By \\ Koichi OGIUE and Ryoichi TAKAGI
}

\section{$\S 1$. Introduction.}

Let $M$ be a Kaehler manifold and denote by $H$ the holomorphic sectional curvature of $M$. We say that $H$ is $\delta$-pinched if there exists a positive constant $c$ such that

$$
\delta c \leqq H \leqq c .
$$

In this paper, we shall prove the following

THEOREM. Let $M$ be a compact irreducible Hermitian symmetric space of rank $r$. Then the holomorphic sectional curvature of $M$ is $\frac{1}{r}$-pinched.

Although it is possible to verify the result for each Hermitian symmetric space one by one by using the curvature tensors given by E. Calabi and E. Vesentini [1], we shall given here a systematic proof.

\section{§ 2. Preliminaries.}

We begin by constructing a compact Hermitian symmetric space. For details, see e.g. [3].

Let $\tilde{g}$ be a complex simple Lie algebra and $\mathfrak{h}$ a Cartan subalgebra of $\tilde{g}$. The dual space of the complex vector space $\mathfrak{h}$ is denoted by $\mathfrak{h}^{*}$. An element $\alpha$ of $\mathfrak{h}^{*}$ is called a root of $(\tilde{g}, \mathfrak{h})$ if there exists a non-zero vector $X_{\alpha}$ in $\tilde{\mathfrak{g}}$ such that

$$
\left[H, X_{\alpha}\right]=\alpha(H) X_{\alpha} \quad \text { for } H \in \mathfrak{h} \text {. }
$$

We denote by $\Delta$ the set of all non-zero roots of $(\tilde{\mathfrak{g}}, \mathfrak{h})$ and put $\mathfrak{g}_{\alpha}=\boldsymbol{C} X_{\alpha}$. Then we have a direct sum decomposition:

$$
\tilde{\mathfrak{g}}=\mathfrak{h}+\sum_{\alpha \in \Delta} \mathfrak{g}_{\alpha} \text {. }
$$

Since the Killing form $K$ of $\tilde{g}$ is non-degenerate on $\mathfrak{h} \times \mathfrak{h}$, for each $\xi \in \mathfrak{h} *$ we can Received July 21, 1980. 
define $H_{\xi} \in \mathfrak{h}$ by

$$
K\left(H, H_{\xi}\right)=\xi(H) \quad \text { for } \quad H \in \mathfrak{h} \text {. }
$$

Put $\mathfrak{h}_{0}=\Sigma_{\alpha \in \Delta} \boldsymbol{R} H_{\alpha}$. Then the dual space $\mathfrak{h}_{0}^{*}$ of $\mathfrak{h}_{0}$ can be considered as a real subspace of $\mathfrak{h}^{*}$. Define an inner product $($,$) on \mathfrak{h}_{0}^{*}$ by

$$
(\xi, \eta)=K\left(H_{\xi}, H_{\eta}\right) \quad \text { for } \xi, \eta \in \mathfrak{h}_{0}^{*} \text {. }
$$

For each $\alpha \in \Delta$ we choose a basis $E_{\alpha}$ of $g_{\alpha}$ so that $\left\{H_{\alpha_{j}}(j=1, \cdots, 1), E_{\alpha}(\alpha \in \Delta)\right\}$ forms Weyl's canonical basis of $\tilde{g}$. Then we have $\left[E_{\alpha}, E_{-\alpha}\right]=H_{\alpha}$, and a Lie algebra $\mathfrak{g}$ defined as follows is a compact real form of $\tilde{g}$ :

$$
\mathfrak{g}=\sum_{\alpha \in \Delta} \boldsymbol{R} \sqrt{-1} H_{\alpha}+\sum_{\alpha \in \Delta} \boldsymbol{R}\left(E_{\alpha}+E_{-\alpha}\right)+\sum_{\alpha \in \Delta} \boldsymbol{R} \sqrt{-1}\left(E_{\alpha}-E_{-\alpha}\right) .
$$

We denote by $\left\{\alpha_{1}, \cdots, \alpha_{l}\right\}$ the fundamental root system of $\tilde{g}$ with respect to a linear ordering in $\mathfrak{h}_{0}^{*}$ (so that $\operatorname{dim}_{C} \mathfrak{h}=l$ ).

Now we fix a simple root $\alpha_{i}(i=1, \cdots, l)$. For simplicity, we put $A_{\alpha}=$ $E_{\alpha}+E_{-\alpha}$ and $B_{\alpha}=\sqrt{-1}\left(E_{\alpha}-E_{-\alpha}\right)$. We define a subset $\Delta_{i}$ of $\Delta$, a subalgebra $\mathfrak{f}_{i}$ of $\mathfrak{g}$ and a subspace $\mathfrak{m}_{i}$ of $\mathfrak{g}$ by

$$
\begin{aligned}
& \Delta_{i}=\left\{\alpha=\sum_{j} m_{j} \alpha_{j} ; m_{i} \geqq 1\right\}, \\
& \mathfrak{f}_{i}=\sum_{\alpha \in \Delta} \boldsymbol{R} \sqrt{-1} H_{\alpha}+\sum_{\alpha \in \Delta^{+}-\Delta_{i}}\left(\boldsymbol{R} A_{\alpha}+\boldsymbol{R} B_{\alpha}\right), \\
& \mathfrak{m}_{i}=\sum_{\alpha \in \Delta_{i}}\left(\boldsymbol{R} A_{\alpha}+\boldsymbol{R} B_{\alpha}\right),
\end{aligned}
$$

where $\Delta^{+}$denotes the set of all positive roots.

Let $G$ be the simply connected Lie group with Lie algebra $g$ and $K_{i}$ the connected Lie subgroup of $G$ with algebra $\mathfrak{f}_{i}$. Let $\pi$ denote the natural projection of $G$ onto a compact homogeneous space $M_{i}=G / K_{i}$ and put $o=\pi\left(K_{i}\right)$. Then we can identify the vector space $\mathfrak{m}_{i}$ with the tangent space $T_{0}\left(M_{i}\right)$ of $M_{i}$ at $o$. It is easily seen that there exists a unique $G$-invariant Riemannian metric $g$ on $M_{i}$ such that $g=-K \mid \mathfrak{m}_{i} \times \mathfrak{m}_{i}$ at $o$. It is known that a compact Riemannian homogeneous space $M_{i}$ obtained as above from a pair $\left(\tilde{g}, \alpha_{i}\right)$ of a complex simple Lie algebra $\tilde{g}$ and a simple root $\alpha_{i}$ becomes a Hermitian symmetric space if and only if the coefficient $m_{i}$ of $\alpha_{i}$ in every $\alpha \in \Delta_{i}$ is equal to 1 and the center $z\left(f_{i}\right)$ of $f_{i}$ is 1-dimensional, and that every compact irreducible Hermitian symmetric space can be obtained in this way.

Hereafter we assume that $M_{i}$ is a Hermitian symmetric space. Then it is known that there exists an element $Z_{0}$ in $z\left(\mathfrak{f}_{i}\right)$ such that the complex structure of $M_{i}$ at $o$ is given by $I=\operatorname{ad} Z_{0} \mid \mathfrak{m}_{i}$ and $I A_{\alpha}=B_{\alpha}, I B_{\alpha}=-A_{\alpha}$ for $\alpha \in \Delta_{i}$. Since $Z_{0} \in z\left(\mathfrak{f}_{i}\right)$, we have 


$$
I \circ A d(k)=A d(k) \circ I \quad \text { for } \quad k \in K_{i} .
$$

Let $\theta^{\alpha}, \theta^{-\alpha}$ be the dual forms of $E_{\alpha}, E_{-\alpha}$. Then we have at $o$

$$
g=2 \sum_{\alpha \in \Delta_{i}} \theta^{\alpha} \theta^{-\alpha},
$$

since $K\left(E_{\alpha}, E_{-\alpha}\right)=-1$. The norm of $X \in \mathfrak{m}_{i}$ is denoted by $|X|$.

\section{§ 3. Proof of Theorem.}

First we state a fundamental lemma without proof.

Lemma (E. Cartan). Let $\mathfrak{a}$ and $\mathfrak{a}^{\prime}$ be two maximal abelian subspaces of $\mathfrak{m}_{i}$. Then

(i) there exists an element $k$ in $K_{i}$ such that $A d(k) \mathfrak{a}=\mathfrak{a}^{\prime}$, and

(ii) $\mathfrak{m}_{i}=\bigcup_{k \in K_{i}} A d(k) \mathfrak{a}$.

The rank $r$ of $M_{i}$ as a symmetric space is, by definition, the common dimension of maximal abelian subspaces of $\mathfrak{m}_{i}$. By a theorem of Harish-Chandra ([2], Lemma 8), there exist $r$ roots $\delta_{1}, \cdots, \delta_{r}$ in $\Delta_{i}$ such that none of $\delta_{i} \pm \delta_{j}$ belong to $\Delta$, which are called strongly orthogonal roots. Thus the space $\mathfrak{a}_{0}$ spanned by $A_{\delta_{1}}, \cdots, A_{\delta_{r}}$ over $\boldsymbol{R}$ is a maximal abelian subspace of $\mathfrak{m}_{i}$. We denote by $R$ the curvature tensor of $\left(M_{i}, g\right)$. Then we have the following formula due to $\mathrm{E}$. Cartan :

$$
R(X, Y) Z=-[[X, Y], Z] \quad \text { for } \quad X, Y, Z \in \mathfrak{m}_{i} .
$$

Put $S=\left\{X \in \mathfrak{m}_{i} ;|X|=1\right\}$. Then, for $X \in S$, the holomorphic sectional curvature $H(X)$ of the plane section spanned by $X$ and $I X$ is given by

$$
\begin{aligned}
H(X) & =g(R(X, I X) I X, X) \\
& =-g([[X, I X], I X], X) \\
& =|[X, I X]|^{2} .
\end{aligned}
$$

We assert that the range of the function $H$ on $S$ coincides with that of $H$ on $S \cap \mathfrak{a}_{0}$. In fact, Lemma implies that, for every $H \in S$, there exists an element $k$ in $K_{i}$ such that $A d(k) X \in S \cap a_{0}$. Therefore from (1) and (3) we have

$$
\begin{aligned}
H(A d(k) X) & =|[A d(k) X, I A d(k) X]|^{2} \\
& =|[A d(k) X, A d(k) I X]|^{2} \\
& =|A d(k)[X, I X]|^{2} \\
& =|[X, I X]|^{2}
\end{aligned}
$$




$$
=H(X) \text {, }
$$

which proves our assertion.

Let $X=\sum_{j=1}^{r} x_{j} A_{\delta_{j}} \in S \cap a_{0}$. Then by (2) we have

$$
\begin{aligned}
1=|X|^{2} & =\sum_{j, k=1}^{r} x_{j} x_{k} g\left(E_{\delta_{j}}+E_{-\delta_{j}}, E_{\delta_{k}}+E_{-\delta_{k}}\right) \\
& =2 \sum_{j=1}^{r} x_{j}^{2},
\end{aligned}
$$

and

$$
\begin{aligned}
{[X, I X] } & =\left[\sum_{j=1}^{r} x_{j} A_{\delta_{j}}, \sum_{k=1}^{r} x_{k} B_{\delta_{k}}\right] \\
& =\sum x_{j}^{2}\left[A_{\delta_{j}}, B_{\delta_{j}}\right] \\
& =\sum x_{j}^{2}\left[E_{\delta_{j}}+E_{-\delta_{j}}, \sqrt{-1}\left(E_{\delta_{j}}-E_{-\delta_{j}}\right)\right] \\
& =-2 \sqrt{-1} \sum x_{j}^{2}\left[E_{\delta_{j}}, E_{-\delta_{j}}\right] \\
& =-2 \sqrt{-1} \sum x_{j}^{2} H_{\delta_{j}} .
\end{aligned}
$$

Hence

$$
\begin{aligned}
|[X, I X]|^{2} & =4\left|\sum x_{j}^{2} H_{\delta_{j}}\right|^{2} \\
& =4 \sum x_{j}^{4}\left(\delta_{j}, \delta_{j}\right) .
\end{aligned}
$$

But by a theorem of C. C. Moore $\left([3]\right.$, p. 362) we have $\left(\delta_{1}, \delta_{1}\right)=\cdots=\left(\delta_{r}, \delta_{r}\right)$. Thus the range of $\mathrm{H}$ is given by

$$
4 r\left(\frac{1}{2 r}\right)^{2}\left(\delta_{1}, \delta_{1}\right) \leqq H \leqq 4\left(\frac{1}{2}\right)^{2}\left(\delta_{1}, \delta_{1}\right),
$$

since $\Sigma x_{j}^{2}=\frac{1}{2}$. Therefore our theorem is proved.

\section{§4. Remark.}

Let $\left(M_{\lambda}, g_{\lambda}\right)$ be a compact irreducible Hermitian symmetric space of rank $r_{\lambda}$ and $H_{\lambda}$ the holomorphic sectional curvature of $\left(M_{\lambda}, g_{\lambda}\right), \lambda=1, \cdots, n$. Assume that $\max H_{1}=\cdots=\max H_{n}$. Then a compact Hermitian symmetric space $\left(M_{1} \times\right.$ $\left.\cdots \times M_{n}, g_{1} \times \cdots \times g_{n}\right)$ of rank $r_{1}+\cdots+r_{n}$ is $\frac{1}{r_{1}+\cdots+r_{n}}$-pinched

\section{References}

[1] Calabi, E. and Vesentini, E., On compact locally symmetric Kaehler manifolds, Ann. of Math. 71 (1960), 472-507.

[2] Harish-Chandra, Representations of semisimple Lie groups VI, Amer. J. Math. 78 
A geometric meaning of the rank of Hermitian symmetric spaces

(1956), 564-628.

[3] Helgason, S., Differential geometry and symmetric spaces, Academic Press, New York, 1962.

[4] Moore, C.C., Compactifications of symmetric spaces II : the Cartan domains, Amer. J. Math. 86 (1964), 358-378.

Tokyo Metropolitan University

University of Tsukuba 